\title{
Improving Support Vector Classification via the Combination of Multiple Sources of Information
}

\author{
Javier M. Moguerza ${ }^{1}$, Alberto Muñoz ${ }^{2}$, and Isaac Martín de Diego ${ }^{2}$ \\ 1 University Rey Juan Carlos, c/ Tulipán s/n, 28933 Móstoles, Spain \\ j.moguerza@escet.urjc.es \\ 2 University Carlos III de Madrid, c/ Madrid 126, 28903 Getafe, Spain \\ alberto.munoz@uc3m.es, ismdiego@est-econ.uc3m.es
}

\begin{abstract}
In this paper we describe several new methods to build a kernel matrix from a collection of kernels. This kernel will be used for classification purposes using Support Vector Machines (SVMs). The key idea is to extend the concept of linear combination of kernels to the concept of functional (matrix) combination of kernels. The functions involved in the combination take advantage of class conditional probabilities and nearest neighbour techniques. The proposed methods have been successfully evaluated on a variety of real data sets against a battery of powerful classifiers and other kernel combination techniques.
\end{abstract}

\section{Introduction}

Support Vector Machines (SVMs) have proven to be a successful tool for the solution of a wide range of classification problems since their introduction in [3]. The method uses as a primary source of information a kernel function $K(i, j)$, where $K$ is Mercer's kernel and $i, j$ represent data points in the sample: By the Representer Theorem (see for instance [16]), SVM classifiers always take the form $f(x)=\sum_{i} \alpha_{i} K(x, i)$. The approximation and generalization capacity of the SVM is determined by the choice of the kernel $K$ [4]. A common way to obtain SVM kernels is to consider a linear differential operator $D$, and choose $K$ as Green's function for the operator $D^{*} D$, where $D^{*}$ is the adjoint operator of $D$ [15]. It is easy to show that $\|f\|^{2}=\|D f\|_{L_{2}}^{2}$. Thus we are imposing smoothing conditions on the solution $f$. However, it is hard to know in advance which particular smoothing conditions to impose for a given data set. Fortunately, kernels are straightforwardly related to similarity (or equivalently distance) measures, and this information is actually available in many data analysis problems.

Nevertheless, using a single kernel may be not enough to solve accurately the problem under consideration. This happens, for instance, when dealing with text mining problems, where analysis results may vary depending on the document similarity measure chosen [9]. Thus, the information provided by a single similarity measure (kernel) may be not enough for classification purposes, and the combination of kernels appears as an interesting alternative to the choice of the 'best' kernel. 
The specific literature on the combination of kernels is rather in its beginnings. A natural approach is to consider linear combinations of kernels. This is the approach followed in [10], and it is based on the solution of a semi-definite programming (SDP) problem to calculate the coefficients of the linear combination. The solution of this kind of optimization problems is computationally very expensive [19]. Another problem regarding this method is the overfitting due to lack of capacity control. A different approach is proposed in [2]. The method, called MARK, builds a classifier (not the specific kernel matrix) by a boosting type algorithm.

In this paper we describe several methods to build a kernel matrix from a collection of kernels for classification purposes. The key idea is to extend the concept of linear combination of kernels to the concept of functional (matrix) combination of kernels.

The paper is organized as follows. Section 2 describes the proposed methods for combining kernels. The experimental setup and results on real data sets are described in section 3. Section 4 concludes.

\section{Methods}

Let $K_{1}, K_{2}, \ldots K_{M}$ be a set of $M$ input kernels defined on a data set $X$, and denote by $K^{*}$ the desired output combination. Let $y$ denote the label vector, where for simplicity $y_{i} \in\{-1,+1\}$ (the extension to the multilabel case is straighforward).

To motivate the discussion, consider the following (functional) weighted sum of the kernels:

$$
K^{*}=\sum_{m=1}^{M} W_{m} \cdot K_{m},
$$

where '.' denotes the element by element product between matrices, and $W_{m}=$ $\left[w_{m}(i, j)\right]$ is a matrix whose elements are nonlinear functions $w_{m}(i, j)$, with $i$ and $j$ data points in the sample. Notice that if $w_{m}(i, j)=\mu_{m}$, where $\mu_{1}, \ldots, \mu_{M}$ are constants, then the method reduces to a simple linear combination of matrices:

$$
K^{*}=\sum_{m=1}^{M} \mu_{m} K_{m}
$$

As mentioned in Section 1, in [10] a method is suggested to learn the coefficients $\mu_{m}$ of the linear combination by solving a semi-definite programming problem. We will refer to this method as SDP.

Taking $\mu_{m}=\frac{1}{M}$, the average of the kernels is obtained. This method will be refered in the following as AKM (Average Kernel Method).

Regarding our proposals, consider the $(i, j)$ element of the matrix $K^{*}$ in $(1)$ :

$$
K^{*}(i, j)=\sum_{m=1}^{M} w_{m}(i, j) K_{m}(i, j) .
$$


Next we will show how to calculate the weighting functions $w_{m}(i, j)$. To this aim, we will make use of conditional class probabilities. Consider the pair $\left(i, y_{i}\right)$ and an unlabeled observation $j$. Given the observed value $j$, define $P\left(y_{i} \mid j\right)$ as the probability of $j$ being in class $y_{i}$. If $i$ and $j$ belong to the same class this probability should be high. Unfortunately this probability is unknown and it has to be estimated. In our proposals we will estimate it by $P\left(y_{i} \mid j\right)=\frac{n_{i j}}{n}$, where $n_{i j}$ is the number of the $n$-nearest neighbours of $j$ belonging to class $y_{i}$. Notice that each kernel induces a different type of neighborhood. Hence, it is advisable to estimate this probability for each kernel representation, that is, for the kernel $K_{m}$ we will estimate the conditional probabilities $P_{m}\left(y_{i} \mid j\right)$.

\subsection{The Probability Weighting Scheme ('ProbWS')}

The first proposed method builds $K^{*}$ by defining $w_{m}(i, j)$ in $(3)$ as:

$$
w_{m}(i, j)=\tau\left(P_{m}\left(y_{i} \mid j\right)+P_{m}\left(y_{j} \mid i\right)\right)^{q},
$$

where $\tau$ is introduced to assure that $\sum_{m} w_{m}(i, j)=1$, and $q$ is a positive constant to control the value of the weights. Within this setting, the weights quantify the relative importance of each kernel: If $i$ and $j$ belong to the same class (say $y_{i}$ ), the proportion of the nearest neighbours of $j$ belonging to $y_{i}$ should be high. So, the method favours the kernel whose induced neighbourhood shows the highest agreement with the data label information.

Given that $K^{*}$ is not necessarily a linear combination of kernels, positive definiteness of $K^{*}$ is not guaranteed. Several solutions have been proposed to face this problem [14]: A first possibility is to replace $K^{*}$ by $K^{*}+\lambda I$, for $\lambda>0$ large enough to make all the eigenvalues of the kernel matrix positive. Another direct approach is to use Multidimensional Scaling to represent the data set in an Euclidean space. Finally, it is also possible to define a new kernel matrix as $K^{* T} K^{*}[17]$.

\subsection{The Exponencial and Polynomial Weighting Scheme Methods}

The next two methods are influenced by the ideas in $[12,5]$ where the variables are weighted according to their relative discrimination power. We make use of similar ideas to raise the weight of kernels with expected good classification performance and, analogously, to diminish the influence of less informative kernels.

Let

$$
\bar{P}\left(y_{i} \mid j\right)=\frac{1}{M} \sum_{m=1}^{M} P_{m}\left(y_{i} \mid j\right),
$$

and

$$
r_{m}(i, j)=\frac{\left(\frac{\bar{P}\left(y_{i} \mid j\right)+\bar{P}\left(y_{j} \mid i\right)}{2}-\frac{P_{m}\left(y_{i} \mid j\right)+P_{m}\left(y_{j} \mid i\right)}{2}\right)^{2}}{\frac{P_{m}\left(y_{i} \mid j\right)+P_{m}\left(y_{j} \mid i\right)}{2}},
$$

where $r_{m}(i, j)$ is designed to measure the ability of kernel $m$ to predict $\bar{P}\left(y_{i} \mid j\right)$ and $\bar{P}\left(y_{j} \mid i\right)$. The value of $r_{m}(i, j)$ will be inversely related to the discrimination 
power of $K_{m}$ with respect to the whole set of kernels: The numerator in (6) approaches zero when the information conveyed by $K_{m}$ tends to be similar to the information collected by the entire set of kernels.

Now, we construct $w_{m}(i, j)$ as a function of $r_{m}(i, j)$. The relative relevance of kernel $K_{m}$ can be evaluated by:

$$
w_{m}(i, j)=\tau \exp \left(q \frac{1}{r_{m}(i, j)}\right) .
$$

We call this method 'exponential weighting scheme' (ExpWS). The parameter $q$ is used to control the influence of $r_{m}(i, j)$ on $w_{m}(i, j)$. If $q=0$, this influence is ignored, and the method reduces to the AKM method. On the other hand, for large values of $q$, changes in $r_{m}$ will be exponentially reflected in $w_{m}$. A different choice to quantify the relative importante of $K_{m}$ is given by:

$$
w_{m}(i, j)=\tau\left(\frac{1}{r_{m}(i, j)}\right)^{q},
$$

where $\tau$ and $q$ play the same role as before. Using $q=1,2$ we have linear and quadratic weighting schemes, respectively. We will label this method as 'polynomical weighting scheme' (PolyWS).

\subsection{The Percentile Methods}

Unlike the previous methods, the techniques introduced in this section do not build a functional (matrix) combination of kernels. Consider the ordered sequence of kernels:

$$
\min _{1 \leq m \leq M} K_{m}(i, j)=K_{[1]}(i, j)<K_{[2]}(i, j)<\ldots<K_{[M]}(i, j)=\max _{1 \leq m \leq M} K_{m}(i, j) .
$$

The two new methods we propose build each element of $K^{*}$ using, respectively, the following formulae:

$$
\begin{gathered}
K^{*}(i, j)=K_{\left[\left(\frac{\bar{P}\left(y_{i} \mid j\right)+\bar{P}\left(y_{j} \mid i\right)}{2}\right) M\right]} \\
K^{*}(i, j)=\frac{K_{\left[\bar{P}\left(y_{i} \mid j\right) M\right]}+K_{\left[\bar{P}\left(y_{j} \mid i\right) M\right]}}{2} .
\end{gathered}
$$

We will denote these methods by 'percentil-in method' and 'percentil-out method', respectively.

If the class probabilities $\bar{P}\left(y_{i} \mid j\right)$ and $\bar{P}\left(y_{i} \mid j\right)$ are high, we can expect a high similarity between $i$ and $j$ and both methods will guarantee a high $K^{*}(i, j)$. If the class probabilities $\bar{P}\left(y_{i} \mid j\right)$ and $\bar{P}\left(y_{i} \mid j\right)$ are both low, $K^{*}(i, j)$ will be also low. 


\subsection{The 'MaxMin' Method}

The next method can be considered as a mixture of the previous combination techniques. The method produces a functional combination of two kernels, namely, the maximun and the minimun of the ordered sequence of kernels:

$$
\begin{array}{r}
K^{*}(i, j)=K_{[M]}(i, j) \frac{\left(\bar{P}\left(y_{i} \mid j\right)+\bar{P}\left(y_{j} \mid i\right)\right)}{2}+ \\
K_{[1]}(i, j) \frac{\left(\bar{P}\left(-y_{i} \mid j\right)+\bar{P}\left(-y_{j} \mid i\right)\right)}{2}
\end{array}
$$

If $i$ and $j$ belong to the same class then the conditional class probabilities $\bar{P}\left(y_{i} \mid j\right)$ and $\bar{P}\left(y_{j} \mid i\right)$ will be high and the method guarantees that $K^{*}(i, j)$ will be large. On the other hand, if $i$ and $j$ belong to diferent classes the conditional class probabilities $\bar{P}\left(y_{i} \mid j\right)$ and $\bar{P}\left(y_{j} \mid i\right)$ will be low and the method will produce a value close to the minimun of the kernels.

\subsection{The 'Pick-out' Method}

This is the limiting case of the 'MaxMin' method. We take $\bar{P}\left(y_{i} \mid j\right)=\bar{P}\left(y_{j} \mid i\right)=1$ if $i$ and $j$ belong to the same class and $\bar{P}\left(y_{i} \mid j\right)=\bar{P}\left(y_{j} \mid i\right)=0$ otherwise.

$$
K^{*}(i, j)= \begin{cases}\max _{1 \leq m \leq M} K_{m}(i, j), & \text { if } i \text { and } j \text { belong to the same class } \\ \underset{1 \leq m \leq M}{1 \leq m} K_{m}(i, j), & \text { if } i \text { and } j \text { belong to different classes }\end{cases}
$$

In this way, if $i$ and $j$ are in the same class, it is guaranteed that $K^{*}(i, j)$ will be the largest possible according to the available information. If $i$ and $j$ belong to different classes, we can expect a low similarity between them, and this is achieved by the choice of the minimum kernel value. This method was first introduced in [13], in the context of classification problems with asymmetric similarity measures.

\section{Experiments}

To test the performance of the proposed methods, a SVM has been trained on several real data sets using the corresponding kernel matrix $K^{*}$. For the ProbWS, ExpWS and PolyWS methods the value of the parameter $q$ has been assigned via cross-validation.

Given a non labelled data point $x, K(x, i)$ has to be evaluated. We can calculate two different values for $K(x, i)$, the first one assumming $x$ belongs to class +1 and the second assumming $x$ belongs to class -1 . For each assumption, all we have to do is to compute the distance between $x$ and the SVM hyperplane and to assign $x$ to the class corresponding to the largest distance from the hyperplane.

In the following, for all the data sets, we will use $80 \%$ of the data for training and $20 \%$ for testing. 
We have compared the proposed methods with the following classifiers: Multivariate Additive Regression Splines (MARS) [6], Logistic Regression (LR), Linear Discriminant Analysis (LDA), k-Nearest Neighbour classification (kNN) and SVMs using a RBF kernel $K_{c}\left(x_{i}, x_{j}\right)=e^{-\left\|x_{i}-x_{j}\right\|^{2} / c}$, with $c=0.5 d$, where $d$ is the data dimension (see [18] for details).

\subsection{Cancer Data Set}

In this section we have dealt with a database from the UCI Machine Learning Repository: the Breast Cancer data set [11]. The data set consists of 683 observations with 9 features each. For this data set we have combined three kernels: a polynomial kernel $K_{1}\left(x_{i}, x_{j}\right)=\left(1+x_{i}^{T} x_{j}\right)^{2}$, a RBF kernel $K_{2}\left(x_{i}, x_{j}\right)=$ $\exp \left(-\left\|x_{i}-x_{j}\right\|^{2}\right)$ and a linear kernel $K_{3}\left(x_{i}, x_{j}\right)=x_{i}^{T} x_{j}$. We have normalized the kernels in order to compare them: $\bar{K}_{i j}=\frac{K_{i j}-\min \left(K_{l s}\right)}{\max \left(K_{l s}\right)-\min \left(K_{l s}\right)}$. The results, averaged over 10 runs, are shown in Table 1.

Table 1. Classification errors for the cancer data.

\begin{tabular}{|c|r|r|r|}
\hline Method & Train error & Test error & Support vectors \\
\hline$K_{1}:$ Polynomial & $0.1 \%$ & $8.6 \%$ & $8.0 \%$ \\
\hline$K_{2}:$ RBF & $0.0 \%$ & $10.2 \%$ & $65.7 \%$ \\
\hline$K_{3}$ :Linear & $2.6 \%$ & $3.6 \%$ & $7.1 \%$ \\
\hline AKM & $1.3 \%$ & $3.8 \%$ & $31.4 \%$ \\
\hline ProbWS & $1.8 \%$ & $3.2 \%$ & $39.6 \%$ \\
\hline ExpWS & $0.0 \%$ & $3.2 \%$ & $90.6 \%$ \\
\hline PolyWS & $2.4 \%$ & $2.9 \%$ & $33.4 \%$ \\
\hline Percentil-in & $1.9 \%$ & $3.4 \%$ & $59.9 \%$ \\
\hline Percentil-out & $1.5 \%$ & $3.5 \%$ & $33.1 \%$ \\
\hline MaxMin & $0.7 \%$ & $2.9 \%$ & $24.5 \%$ \\
\hline Pick-out & $1.7 \%$ & $2.9 \%$ & $11.4 \%$ \\
\hline kNN & $2.79 \%$ & $3.6 \%$ & $18.3 \%$ \\
\hline MARK-L & $0.0 \%$ & $11.7 \%$ & \\
\hline MARS & $2.7 \%$ & $3.2 \%$ & \\
\hline LDA & $3.8 \%$ & $4.4 \%$ & $61.4 \%$ \\
\hline LR & $13.1 \%$ & $13.1 \%$ & $49.5 \%$ \\
\hline SDP & $0.0 \%$ & $6.6 \%$ & \\
\hline SVM & $0.0 \%$ & $4.4 \%$ & \\
\hline
\end{tabular}

The Pick-out, MaxMin and PolyWS methods show the best overall performance. All our combination methods provide better results than the SVM with a single kernel, using usually significantly less support vectors. The standard deviation of the test error (over 10 runs) was below $1 \%$ for all the studied methods.

It is well known that the choice of kernel parameters is often critical for the good performance of SVMs. Combining kernels provides a solution that minimizes the effect of a bad parameter choice. Next we illustrate this situation 
using a battery of RBF kernels on the cancer data set. Let $\left\{K_{1}, \ldots, K_{12}\right\}$ be a set of RBF kernels with parameters $c=0.1,1,10,20,30,40,50,60,70,80,90,100$ respectively. Table 2 shows the performance of the proposed methods when combining all these kernels. Again, the results have been averaged over 10 runs.

Table 2. Classification errors for the cancer data using a battery of RBF kernels.

\begin{tabular}{|c|r|r|r|}
\hline Method & Train error & Test error & Support vectors \\
\hline Best RBF & $2.3 \%$ & $2.6 \%$ & $13.6 \%$ \\
\hline Worst RBF & $0.0 \%$ & $24.8 \%$ & $73.5 \%$ \\
\hline AKM & $1.6 \%$ & $2.9 \%$ & $21.6 \%$ \\
\hline ProbWS & $1.4 \%$ & $2.9 \%$ & $19.2 \%$ \\
\hline ExpWS & $1.6 \%$ & $2.8 \%$ & $21.4 \%$ \\
\hline PolyWS & $0.1 \%$ & $2.9 \%$ & $31.6 \%$ \\
\hline Percentil-in & $1.9 \%$ & $2.6 \%$ & $7.7 \%$ \\
\hline Percentil-out & $1.7 \%$ & $2.5 \%$ & $9.5 \%$ \\
\hline MaxMin & $1.9 \%$ & $2.6 \%$ & $9.0 \%$ \\
\hline Pick-out & $2.7 \%$ & $3.2 \%$ & $7.7 \%$ \\
\hline MARK-L & $0.0 \%$ & $3.5 \%$ & $18.3 \%$ \\
\hline SDP & $0.0 \%$ & $3.1 \%$ & $39.1 \%$ \\
\hline
\end{tabular}

The Percentil-out method improves the best RBF kernel under consideration while the MaxMin and Percentil-in methods show a similar performance to that of the best RBF. In particular, the results provided by the combination methods are not degraded by the inclusion of kernels with a bad generalization performance.

\subsection{A Handwritten Digit Recognition Problem}

The experiment in this section is a binary classification problem: the recognition of digits ' 7 ' and ' 9 ' from the Alpaydin and Kaynak database [1]. The data set is made up by 1128 records, represented by $32 \times 32$ binary images. We have employed three different methods to specify features in order to describe the images. The first one is the $4 \times 4$ method: features are defined as the number of ones in each of the 64 squares of dimension $4 \times 4$. The second method was introduced by Frey and Slate [7]: 16 attributes are derived from the image, related to the horizontal/vertical position, width, height, etc. The last method under consideration was designed by Fukushima and Imagawa [8]: features are defined as a collection of 12 different representations in a $4 \times 4$ square. This is a typical example with several different sources of information and probably complementary. We have used these representations to calculate three kernels from the Euclidean distance. Classifier performance for all the methods is tabulated in Table 3 . We have taken the $4 \times 4$ representation to train $\mathrm{kNN}$, MARS, LR and LDA methods. The Percentil-in and Percentil-out methods achieve the best results on classification. Furthermore, the MaxMin, Pick-out and ExpWS combinations improve the results obtained using the rest of the techniques except 
$\mathrm{kNN}$. The excellent performance of $\mathrm{kNN}$ may be due to the fact that the result in the table has been obtained using only the best digit codification $(4 \times 4)$. When the other codification methods are used, the error of $\mathrm{kNN}$ increases up to $1.7 \%$.

Table 3. Classification errors for the handwritten digit data set.

\begin{tabular}{|c|r|r|r|}
\hline Method & Train error & Test error & Support vectors \\
\hline $4 \times 4$ & $0.0 \%$ & $3.6 \%$ & $3.6 \%$ \\
\hline Frey-Slate & $5.5 \%$ & $11.1 \%$ & $9.8 \%$ \\
\hline Fukushima & $0.0 \%$ & $4.5 \%$ & $7.0 \%$ \\
\hline AKM & $0.0 \%$ & $4.5 \%$ & $13.1 \%$ \\
\hline ProbWS & $0.0 \%$ & $4.5 \%$ & $13.1 \%$ \\
\hline ExpWS & $0.5 \%$ & $3.3 \%$ & $15.1 \%$ \\
\hline PolyWS & $0.5 \%$ & $3.6 \%$ & $4.6 \%$ \\
\hline Percentil-in & $0.0 \%$ & $1.1 \%$ & $10.9 \%$ \\
\hline Percentil-out & $0.0 \%$ & $1.1 \%$ & $32.8 \%$ \\
\hline MaxMin & $0.0 \%$ & $1.9 \%$ & $34.2 \%$ \\
\hline Pick-out & $0.0 \%$ & $3.1 \%$ & $16.9 \%$ \\
\hline kNN & $0.0 \%$ & $0.6 \%$ & $13.0 \%$ \\
\hline MARK-L & $0.0 \%$ & $4.2 \%$ & \\
\hline MARS & $0.1 \%$ & $3.9 \%$ & \\
\hline LDA & $0.4 \%$ & $5.0 \%$ & $6.2 \%$ \\
\hline LR & $0.0 \%$ & $3.6 \%$ & \\
\hline SDP & $0.0 \%$ & $3.6 \%$ & \\
\hline
\end{tabular}

\section{Conclusions}

In this work we have proposed several techniques for the combination of kernels within the context of SVM classifiers. The suggested methods compare favorably to other well established classification techniques and also to other kernel combining techniques in a variety of real data sets. Within the group of the combining techniques proposed in this paper, there is not an overall better method. Further research will focus on the theoretical properties of the methods and extensions.

\section{Acknowledgments}

This work was partially supported by Spanish grants BEC2000-0167 (DGICYT), TIC2003-05982-C05-05 (MCyT) and PPR-2003-42 (URJC).

\section{References}

1. E. Alpaydin and C. Kaynak. Cascading Classifiers. Kybernetika 34(4):369-374, 1998.

2. K. Bennett, M. Momma, and J. Embrechts. MARK: A Boosting Algorithm for Heterogeneous Kernel Models. Proceedings of SIGKDD International Conference on Knowledge Discovery and Data Mining, 2002. 
3. C. Cortes and V. Vapnik. Support Vector Networks. Machine Learning, 20:273-297, 1995.

4. N. Cristianini and J. Shawe-Taylor. An Introduction to Support Vector Machines. Cambridge University Press, 2000.

5. C. Domeniconi, J. Peng and D. Gunopulos Adaptive Metric Nearest Neighbor Classification. Proc. of IEEE Conf. on CVPR, 517-522, 2000.

6. J. Friedman. Multivariate adaptative regression splines (with discussion). Annals of Statistics, 19 (1):1-141, 1991.

7. P.W. Frey and D.J. Slate. Letter Recognition Using Holland-Style Adaptive Classifiers. Machine Learning, 6 (2):161-182, 1991.

8. K. Fukushima and T. Imagawa. Recognition and segmentation of connected characters with selective attention. Neural Networks, 6:33-41, 1993.

9. T. Joachims. Learning to Classify Text using Support Vector Machines. Kluwer, 2002.

10. G.R.G. Lanckriet, N. Cristianini, P. Barlett, L. El Ghaoui and M.I. Jordan. Learning the kernel matrix with semi-definite programming. Journal of Machine Learning Research, 5:27-72, 2004.

11. O.L. Mangasarian and W.H. Wolberg. Cancer diagnosis via linear programming. SIAM News, 23 (5):1-18, 1990.

12. A. Muñoz and T. Villagarcía. Unsupervised neural networks for variable selection with mixed covariates. Analyse Multidimensionelle des Donnes, CSIA Ceresta, 217-227, 1998.

13. A. Muñoz, I. Martín de Diego and J.M. Moguerza. Support Vector Machine Classifiers for Assymetric Proximities. Proc. ICANN (2003), LNCS, Springer, 217-224.

14. E. Pekalska, P. Paclík and R.P.W. Duin. A Generalize Kernel Approach to Dissimilarity-based Classification. Journal of Machine Learning Research, Special Issue on Kernel Methods 2 (2):175-211, 2002.

15. T. Poggio and F. Girosi. Networks for Approximation and Learning. Proceedings of the IEEE, 78(10):1481-1497, 1990.

16. B. Schölkopf, R. Herbrich, A. Smola and R. Williamson. A Generalized Representer Theorem. NeuroCOLT2 TR Series, NC2-TR2000-81, 2000.

17. B. Schölkopf, S. Mika, C. Burges, P. Knirsch, K. Müller, G. Rätsch and A. Smola. Input Space versus Feature Space in Kernel-based Methods. IEEE Transactions on Neural Networks 10 (5):1000-1017, 1999.

18. B. Schölkopf, J.C. Platt, J. Shawe-Taylor, A.J. Smola and R.C. Williamson. Estimating the Support of a High Dimensional Distribution. Neural Computation, 13(7):1443-1471, 2001.

19. L. Vandenberghe and S. Boyd. Semidefinite programming. SIAM Review, 38(1):4995, 1996. 